\title{
Characterization and Reduction of Noise in Dynamic PET Data Using Masked Volumewise Principal Component Analysis
}

\author{
Per-Edvin Svensson ${ }^{1}$, Johan Olsson ${ }^{1}$, Fredrik Engbrant ${ }^{2}$, Ewert Bengtsson ${ }^{1}$, and Pasha Razifar ${ }^{1,2}$ \\ ${ }^{I}$ Centre for Image Analysis, Uppsala University, Uppsala, Sweden; and ${ }^{2}$ GEMS PET Systems AB, Uppsala Applied Science \\ Laboratory, GE Healthcare, Uppsala, Sweden
}

\begin{abstract}
Masked volumewise principal component (PC) analysis (PCA) is used in PET to distinguish structures that display different kinetic behaviors after administration of a tracer. When masked volumewise PCA was introduced, one article proposed noise prenormalization because of temporal and spatial variations of the noise between slices. However, the noise prenormalization proposed in that article was applicable only to datasets reconstructed using filtered backprojection (FBP). The study presented in this article aimed at developing a new noise prenormalization that is applicable to datasets regardless of whether they were reconstructed with FBP or an iterative reconstruction algorithm, such as ordered-subset expectation maximization (OSEM). Methods: A phantom study was performed to investigate differences in the expectation values and SDs of datasets reconstructed with FBP and OSEM. A novel method, higher-order PC noise prenormalization, was suggested and evaluated against other prenormalization methods on clinical datasets. Results: Masked volumewise PCA of data reconstructed with FBP was much more dependent on an appropriate prenormalization than was analysis of data reconstructed with OSEM. Higher-order PC noise prenormalization showed an overall good performance with both FBP and OSEM reconstructions, whereas the other prenormalization methods performed well with only 1 of the 2 methods. Conclusion: Higher-order PC noise prenormalization has potential for improving the results from masked volumewise PCA on dynamic PET datasets independent of the type of reconstruction algorithm.
\end{abstract}

Key Words: positron emission tomography; masked volumewise principal component analysis; dynamic PET; noise

J Nucl Med Technol 2011; 39:27-34

DOI: $10.2967 /$ jnmt.110.077347

\section{$\mathbf{P}$} principle of annihilation coincidence detection (1). PET is

\footnotetext{
Received Mar. 18, 2010; revision accepted Oct. 7, 2010.

For correspondence or reprints contact: Pasha Razifar, Uppsala Applied Science Laboratory (UASL), GE Healthcare, Husbyborg, Uppsala, Up, Sweden 75228.

E-mail: pasha.razifar@ge.com

COPYRIGHT @ 2011 by the Society of Nuclear Medicine, Inc.
}

used in medical and research applications to visualize the physiologic interactions between an administered tracer and targets of interest or other tissues.

A common approach to reducing noise in dynamic PET data is to sum image volumes within a given time interval. The drawback of this approach is that the differences in the kinetic behavior of the tracer in different tissues are hard to discern.

Multivariate analysis methods, such as masked volumewise principal component (PC) analysis (PCA), have been used on data from dynamic PET studies to retrieve weighted sums of image volumes. These components can separate regions where the tracer has different kinetic behaviors $(2,3)$. In the masked volumewise PCA method, analysis is applied to a dataset consisting of whole volumes of dynamic PET data. Before application of PCA, pixels outside the scanned object are removed and the remaining pixels are prenormalized to compensate for different levels of noise at different times during the scan. This noise prenormalization helps PCA to better separate noise from signal, thus better separating the different tracer kinetics and reducing the noise in the resulting PCs.

However, the background noise prenormalization associated with the masked volumewise PCA method is applicable only to datasets reconstructed with filtered backprojection (FBP), because the noise prenormalization estimates the average SD of each slice by calculating the sample SD of the background. Iterative reconstruction methods, such as ordered-subset expectation maximization (OSEM), have much lower noise in regions with low count density and therefore a significantly lower SD in the background (4). Therefore, no noise prenormalization is currently used when masked volumewise PCA is performed on datasets reconstructed with OSEM.

The aim of this study was to reduce noise in dynamic PET datasets, reconstructed with either FBP or OSEM, using masked volumewise PCA.

\section{MATERIALS AND METHODS}

\section{Phantom Study}

A phantom study was performed to investigate the differences in expectation value and SD of PET image volumes reconstructed with FBP and OSEM. 
A phantom with 2 cylindric inserts was used, both 15 $\mathrm{mm}$ in diameter. The insert in the upper part of the gantry was filled with $223 \mathrm{kBq}$ of ${ }^{18} \mathrm{~F}$ per milliliter, the insert in the left part of the gantry with $73 \mathrm{kBq}$ of ${ }^{18} \mathrm{~F}$ per milliliter, and the rest of the phantom with water. The duration of the emission scan was $90 \mathrm{~min}$.

The study was performed on an eXplore VISTA smallanimal PET scanner (GE Healthcare). The unit contained 2 rings of 18 phosphor sandwich (phoswich) detector modules capable of acquiring 3-dimensional data with an axial field of view of $48 \mathrm{~mm}$ and an effective transaxial field of view of $67 \mathrm{~mm}$ (5).

Acquired data were reconstructed to 2 different datasets, using Fourier rebinning followed by 2-dimensional OSEM (2 iterations and 16 subsets) as well as FBP with ramp and Hanning filters, both using 5-min frames. The dimensions of the reconstructed datasets were $175 \times 175 \times 61 \times 18,175 \times$ 175 pixels with $0.3875-\mathrm{mm}$ sides per transaxial slice, 61 slices with $0.775-\mathrm{mm}$ spacing, and 18 frames of 5 min each.

Three circular regions of interest (ROIs) of equal size were calculated and drawn, one for each insert and one in a region where no radioactive substance was present (Fig. 1). Each ROI had a diameter of $11 \mathrm{~mm}$, which gave 633 pixels per ROI. To avoid most of the spillover effects, the diameter was reduced by $4 \mathrm{~mm}$. This value was chosen because the spatial resolution measured in full width at half maximum is less than $2 \mathrm{~mm}$ for the eXplore VISTA scanner.

The arithmetic mean and sample SD within the ROIs were calculated for each slice and frame for data reconstructed with FBP and OSEM. Furthermore, the arithmetic means of these estimates for all ROIs at a given slice and frame were calculated.

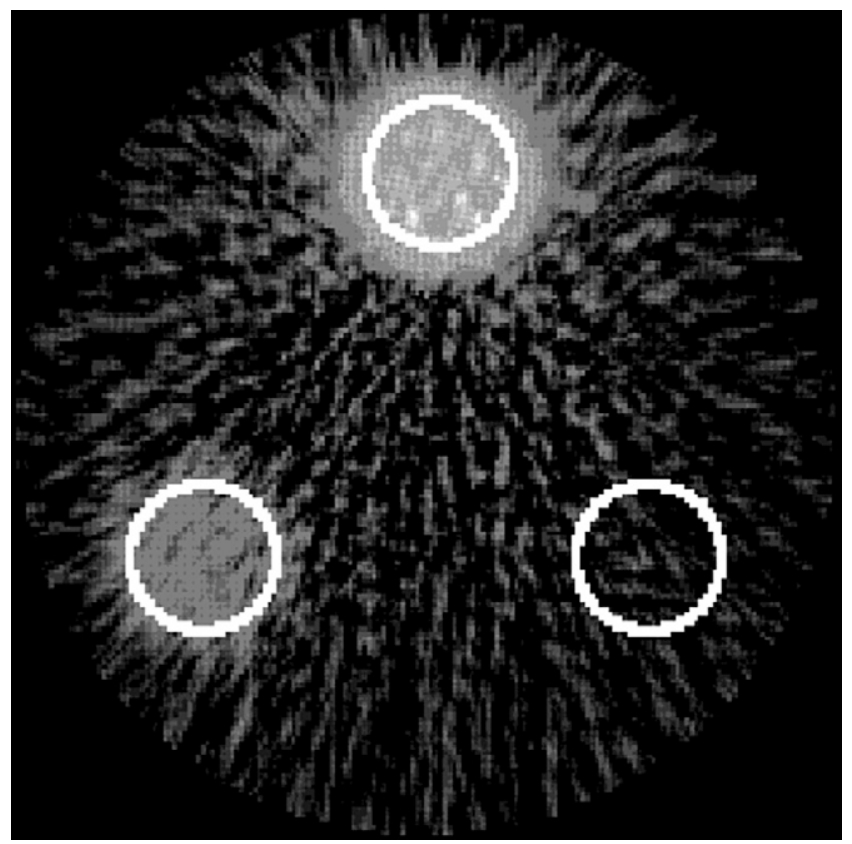

FIGURE 1. Cross-section of phantom reconstructed with FBP. The 3 ROls are outlined in white.

\section{Data Analysis}

All the presented clinical data were analyzed using masked volumewise PCA with the prenormalization and PCA methods described in the following sections. The data in the clinical study were masked using a previously described masking method (6). In the phantom study, a volume of interest (VOI) was created using a simple cylinder of the size of the phantom. Because the phantom was perfectly symmetric and had known dimensions, no image analytic methods were required to produce a VOI.

\section{Prenormalizations}

Two methods are commonly used to prenormalize data before ordinary PCA. The first is to remove the mean of each observation, in this article referred to as removal-ofmean prenormalization. The second is to use standardized variables, which are acquired by removing the mean and dividing each observation by its SD.

In PET image volumes reconstructed with FBP, the background contains a large amount of noise. In 2-dimensional reconstructions of PET data, each slice tends to have varying levels of noise. Because PCA cannot separate variance due to signal from variance due to noise, it is desirable to have each slice scaled with the SD of the noise to get unit noise variance in each slice.

Background noise prenormalization uses a mask to separate the background from the object to estimate the $\mathrm{SD}$ of the background in each slice. The actual prenormalization calculations are similar to those used in standardized variable prenormalization except that the SD of the background in each slice is used instead of the SD of the whole signal in each frame. This method is used only on PET data reconstructed with FBP, because image volumes reconstructed with OSEM have values close to zero in the background (2).

\section{Higher-Order PC Noise Prenormalization}

Higher-order PC noise prenormalization is a novel method presented for the first time in this article. Much like background noise prenormalization, it estimates the SD of the noise in each slice, which is used for prenormalizing the slices.

Because most of the expectation value in dynamic PET data is accounted for by the lower-order masked volumewise PCs, the SD of the noise can be approximated from the reconstruction of higher-order PCs.

Higher-order PC noise prenormalization has 3 steps. The first is to perform masked volumewise PCA on the whole field of view or the VOI without any prenormalization. The second step is to reconstruct the dataset using only the higher-order masked volumewise PCs and estimate the SD in each slice in the reconstructed data. The third step is to calculate the prenormalized variables using the equation

$$
z_{i k}=\frac{\left(x_{i k}-\bar{x}_{i}\right)}{s_{w}} .
$$


$x_{i k}$ is the $k$ th sample in frame $i, \bar{x}_{i}$ is the arithmetic mean in frame $i$, and $s_{w}$ is the sample SD in slice $w$ where the sample $x_{i k}$ is located within the reconstruction of higherorder PCs. The whole procedure is illustrated in Figure 2.

\section{Reconstruction from Selected PCs}

Because the signal in PET datasets is temporally correlated and the noise is not, masked volumewise PCA can be used to reduce the dimensionality of datasets. In the space spanned by the masked volumewise PCs, the signal is described mostly by lower-order masked volumewise PCs whereas noise is described by higher-order masked volumewise PCs. It is therefore useful to be able to separate data spanned by the lower-order masked volumewise PCs from data spanned by the higher-order masked volumewise $\mathrm{PCs}$ in the original frame space.

Because PCA and masked volumewise PCA with nonmasked data changes merely the basis of the prenormalized data, no quantitative information is lost during the masked volumewise PCA and the full original dataset or parts of it can be reconstructed from the masked volumewise PCs.

A PET dataset with $p$ frames is defined as

$$
X=\left[\mathrm{X}_{1}, \mathrm{X}_{2}, \ldots, \mathrm{X}_{p}\right]^{\mathrm{T}} .
$$

Prenormalizing this matrix yields the matrix $Z$. To remove all but the $\mathrm{k}$ lowest-order $\mathrm{PCs}$ from $\mathrm{Z}, \mathrm{Z}$ is projected onto the corresponding eigenvectors retrieved from PCA of $\mathrm{Z}$. Instead of representing this signal as $p$ frames, one can represent it by $k \mathrm{PCs}$, by projecting the data onto the eigenvectors retrieved from PCA of the data. The PCs are calculated from

$$
\mathrm{Y}=\mathrm{EZ}=\left[\mathrm{y}_{1}, \mathrm{y}_{2}, \ldots, \mathrm{y}_{k}\right]^{\mathrm{T}}
$$

where $\mathrm{Z}$ is the prenormalized matrix $\mathrm{X}$ and $\mathrm{E}$ is the eigenvector matrix retrieved from the PCA. To project a selected number of PCs back to frame space, all unwanted PCs in $\mathrm{Y}$ are set to zero, creating the new matrix $\tilde{\mathrm{Y}}$. The prenormalized signal in frame space is then calculated as

$$
\tilde{\mathrm{Z}}=\mathrm{E}^{-1} \tilde{\mathrm{Y}}=\mathrm{E}^{\mathrm{T}} \tilde{\mathrm{Y}} .
$$

The inverse prenormalization is then performed on $\tilde{Z}$, creating the modified input matrix $\tilde{X}$. A flow chart of the procedure is shown in Figure 3.

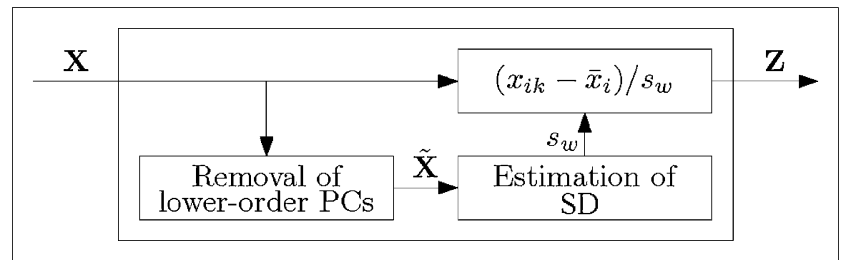

FIGURE 2. Schematic of higher-order PC noise prenormalization.

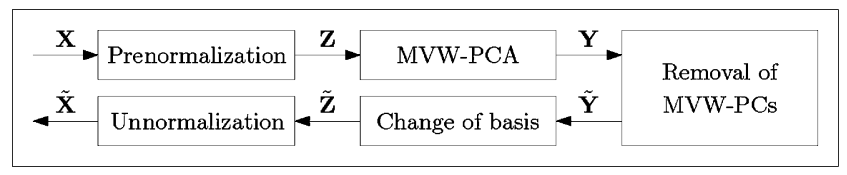

FIGURE 3. Removal of masked volumewise (MVW) PCs from $X$.

\section{Clinical Study to Trim a Higher-Order PC Noise Prenormalization Parameter}

To determine the optimal number of masked volumewise PCs used for performing the higher-order PC noise prenormalization, the sample SDs in different reconstructions were calculated. These estimates were then compared with the sample SD of the background in a dataset reconstructed with FBP.

The mean squared error (MSE) was used to retrieve a quantitative measure of the differences between the estimates from the reconstructions and the estimate from the background. Because PCA picks the same eigenvectors no matter the scale of the input data, a multiplication of the prenormalization coefficients by a scalar will not affect the PCA results. Therefore, each estimate from the reconstructions was multiplied by the scalar value that minimized the MSE to the estimate from the background, to get a fair comparison of MSEs. These comparisons were made between 2 clinical datasets reconstructed with FBP, where the first dataset was retrieved from a full-body study performed with ${ }^{18} \mathrm{~F}$-FDG and the second was a brain study performed with ${ }^{11} \mathrm{C}$-Pittsburgh compound $\mathrm{B}$.

\section{Masked Volumewise PCA}

PCA is a method that explains the variance-covariance structure through linear combinations of the original variables (7). Each linear combination, known as PC, is picked in such a way that it maximizes the variance, which is the same as minimizing the MSE, under the constraint that the norm of its weight vector equals 1 and that the new PC is uncorrelated to any previous PCs (8). The weight vectors are the eigenvectors of the covariance matrix of the multivariate data and are usually calculated by singular value decomposition.

Because it is common that only a limited part of a dataset contains the object, the dataset can be masked to include only data within this VOI. This procedure reduces memory use and computation time and has an advantage in that the directions of the eigenvectors are dependent only on data inside the VOI and are not influenced by noise or other disturbing signals in the background. Masked volumewise PCA is performed in several steps: A mask representing the scanned object is created using transmission images either from PET or, if a PET/CT study is performed, from CT. The mask is used to extract the object from the background and can also be used to perform background noise prenormalization. PCA is performed on the data within the VOI representing the object. PCs created with masked volumewise PCA are referred to as masked volumewise PCs (2). To be 
FIGURE 4. Illustration of masked volumewise PCA procedure.

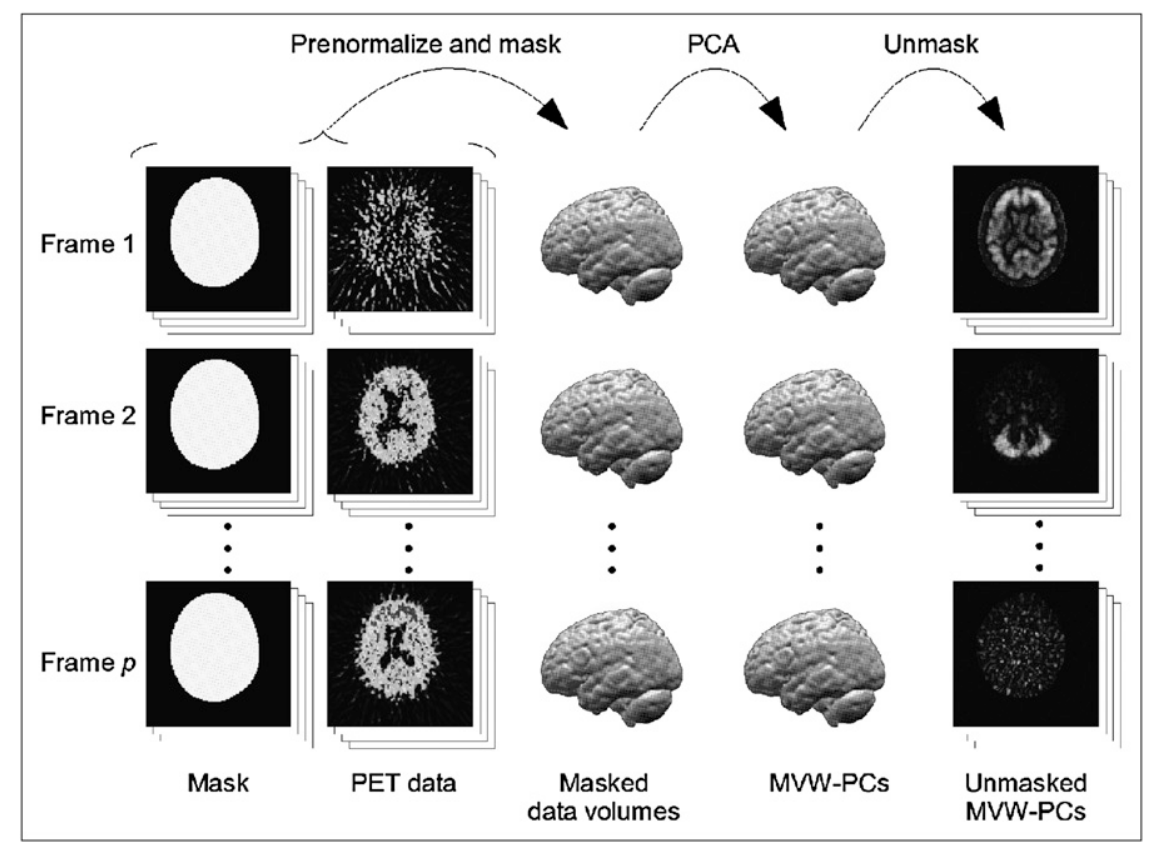

viewed, the masked volumewise PCs are placed in a matrix of the same size as the input data, creating the unmasked masked volumewise PCs seen in Figure 4.

\section{Clinical Study for Evaluating Higher-Order PC Noise Prenormalization}

To evaluate the higher-order PC noise prenormalization, we used a clinical dynamic PET dataset from a previous study (3). This dataset contained 14 frames and was acquired from a full-body study performed with ${ }^{11} \mathrm{C}$-metomidate. Reconstructed datasets generated using both FBP and OSEM were available.

Two VOIs were selected in the OSEM reconstructed data: one over an adrenal gland and another over the stomach.

Both the FBP and the OSEM datasets were prenormalized with the removal-of-mean, standardized-variable, background, and higher-order PC prenormalizations, creating 8 new datasets. Masked volumewise PCA was applied to all datasets, and reconstructions were performed with masked volumewise PC 1; with masked volumewise PC 1 and masked volumewise PC 2; with masked volumewise PC 1, masked volumewise PC 2, and masked volumewise PC 3; and so on until all but the highest-order masked volumewise PC (masked volumewise PC 14) were used in a reconstruction. In this way, 104 datasets $(8 \times 13=104)$ were created.

Usually, when a VOI is drawn within an organ, the volume is not completely homogeneous. There are variations in scale between the time-activity curves, yet it can often be assumed that they all share a similar kinetic behavior. Since the fact that time-activity curves differ only in scale can be described by a single component, masked volumewise PCA was performed on the VOI to find the masked volumewise PC that optimally represented the time-activity curve within the VOI in a mean square sense. This optimal component was reconstructed and referred to

FIGURE 5. Arithmetic mean (A-C) and sample SD (D-F) for FBP (dashed line) and OSEM (solid line) over time in the 3 ROls: high-activity ROI (A and D), lowactivity $R O I$ ( $B$ and $E$ ), and no-activity $\mathrm{ROI}(\mathrm{C}$ and $\mathrm{F})$.

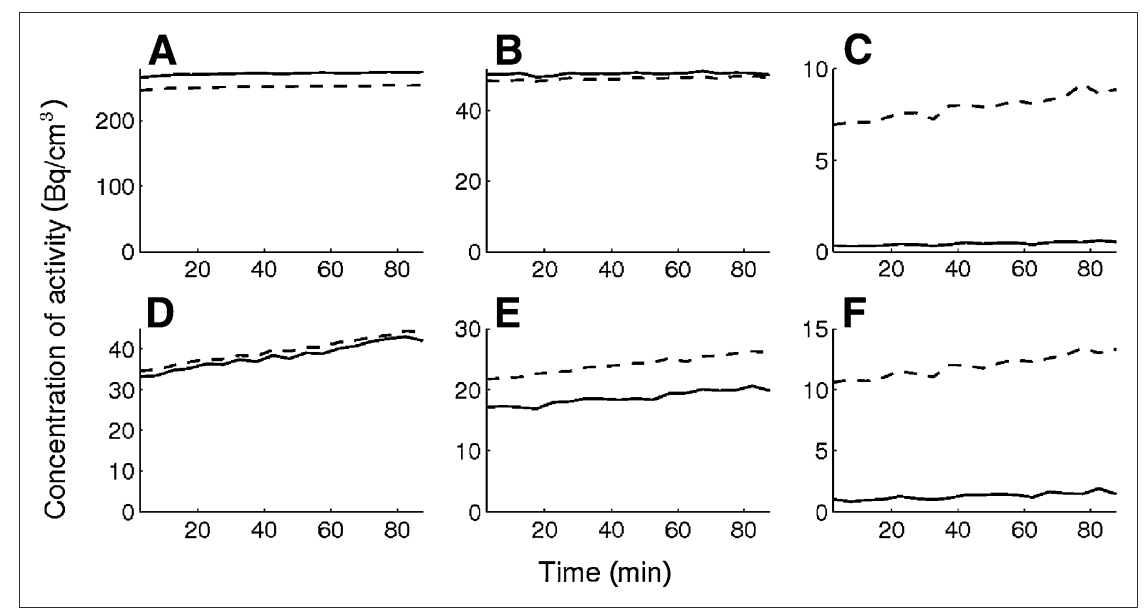




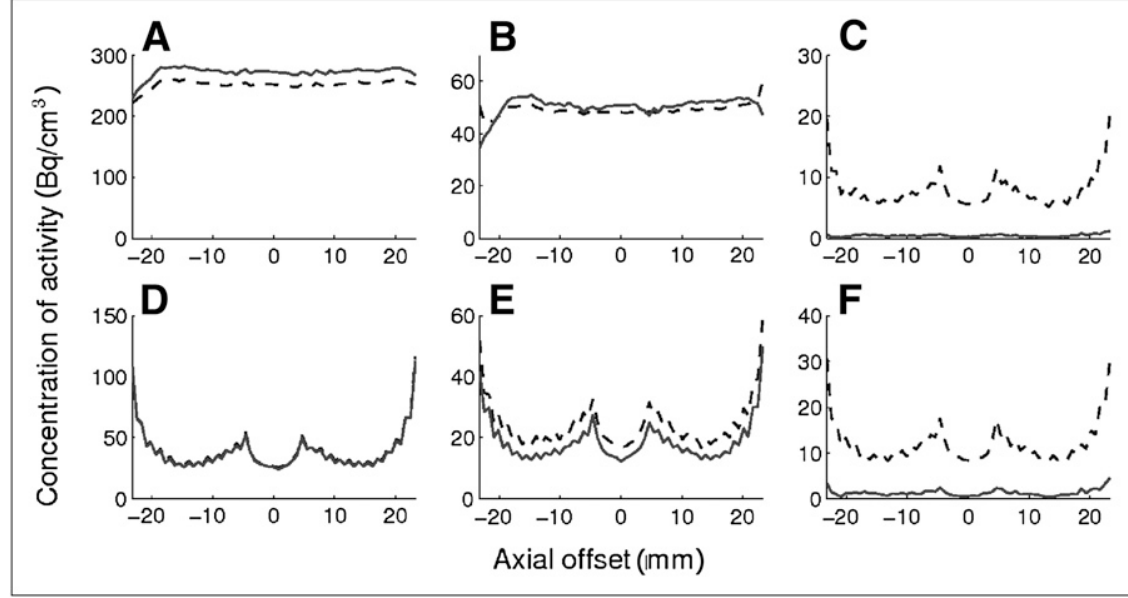

FIGURE 6. Arithmetic mean $(\mathrm{A}-\mathrm{C})$ and sample SD (D-F) for FBP (dashed line) and OSEM (solid line) for different axial offsets in the 3 ROls: high-activity ROI (A and D), low-activity ROI (B and E), and no-activity $\mathrm{ROI}(\mathrm{C}$ and $\mathrm{F})$. as $\hat{x}$. To measure the deviation between a dimensionreduced dataset $\tilde{x}$ and the optimal component $\hat{x}$, the MSE between the samples was calculated as

$$
\operatorname{MSE}_{\hat{x}}=\frac{1}{p \cdot N_{\mathrm{VOI}}} \sum_{n=1}^{p} \sum_{i=1}^{N_{\mathrm{VOI}}}(\hat{x}[i, n]-\tilde{x}[i, n])^{2},
$$

where $p$ is the number of frames and $N_{\text {VOI }}$ is the number of samples within the VOI.

\section{RESULTS}

\section{Comparison of Data Reconstructed with FBP and OSEM}

The arithmetic mean and sample SD for the 3 ROIs over time (different frames) are shown in Figure 5. The arithmetic mean over all slices for each given frame was calculated to get more stable results.

OSEM had a slightly higher arithmetic mean in the highand low-activity ROIs than did FBP, whereas the arithmetic mean in the no-activity ROI was far lower for OSEM than for FBP. Compared with FBP, OSEM also showed a highly decreased sample SD in the low- and no-activity ROIs. Apart from the differences between FBP and OSEM, one can notice that the arithmetic mean is nearly constant, whereas the sample SD shows a positive trend over time.
The corresponding measurements for different axial offsets (different slices) are shown in Figure 6. In this case, the arithmetic means over all frames for each given slice were calculated to get more stable results.

Other general observations were that the outer slices, close to the opening of the gantry, had lower arithmetic means than the slices near the center because of detection of counts outside the field of view. The curve representing the sample SD had a characteristic shape with high values near the edges and 2 peaks at $\pm 4.65 \mathrm{~mm}$ (slices 25 and 37 ). The curves for the arithmetic mean in the no-activity ROI had a similar shape. One can also notice that odd slices tend to have a slightly higher sample SD than the neighboring even slices, resulting in a jagged appearance.

\section{Clinical Study to Trim a Higher-Order PC Noise Prenormalization Parameter}

A comparison between the sample SD of the background and the scaled sample SD of 4 different reconstructions from higher-order PCs is shown in Figure 7.

The deviation from the SD of the reconstructions compared with the background noise was measured by calculation of the MSE for up to 13 masked volumewise PCs. The results are shown in Figure 8. Reconstruction without the first or the first 2 masked volumewise PCs in the higher-

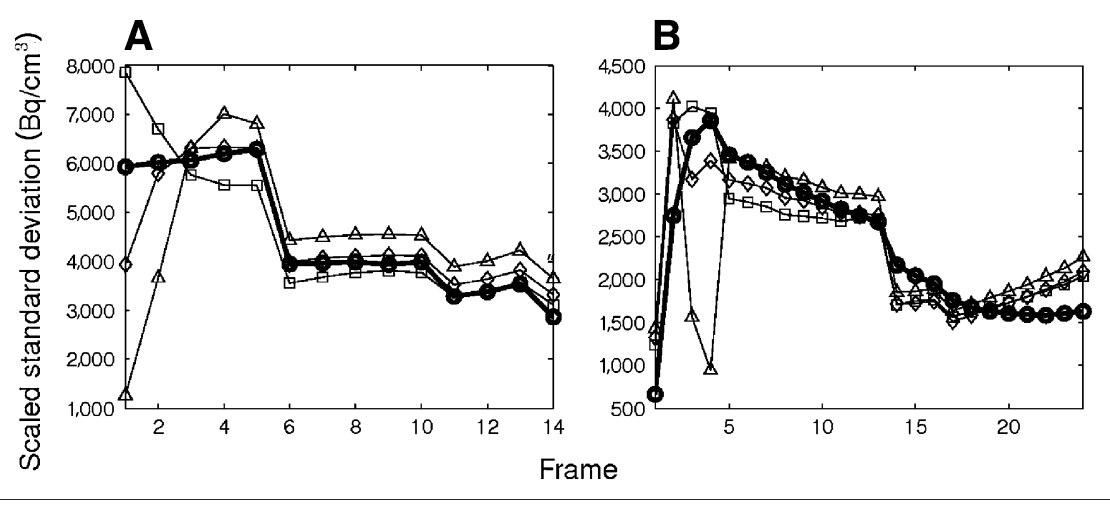

FIGURE 7. Scaled plots of SD used by higher-order $\mathrm{PC}$, from full-body study using ${ }^{18} \mathrm{~F}-\mathrm{FDG}(\mathrm{A})$ and brain study using ${ }^{11} \mathrm{C}-\mathrm{Pittsburgh}$ compound $\mathrm{B}(\mathrm{B})$. Higherorder PC $1(\square)$, higher-order PC $2(\diamond)$, and higher-order PC $3(\Delta)$ are compared with background noise $(\bigcirc)$. Index of higherorder PC corresponds to number of removed masked volumewise PCs in prenormalization step. 
FIGURE 8. MSE between SD of background noise and scaled SD used by higher-order PC noise prenormalization: result from full-body study $(A)$ and result from brain study (B). Logarithmic scale is used.

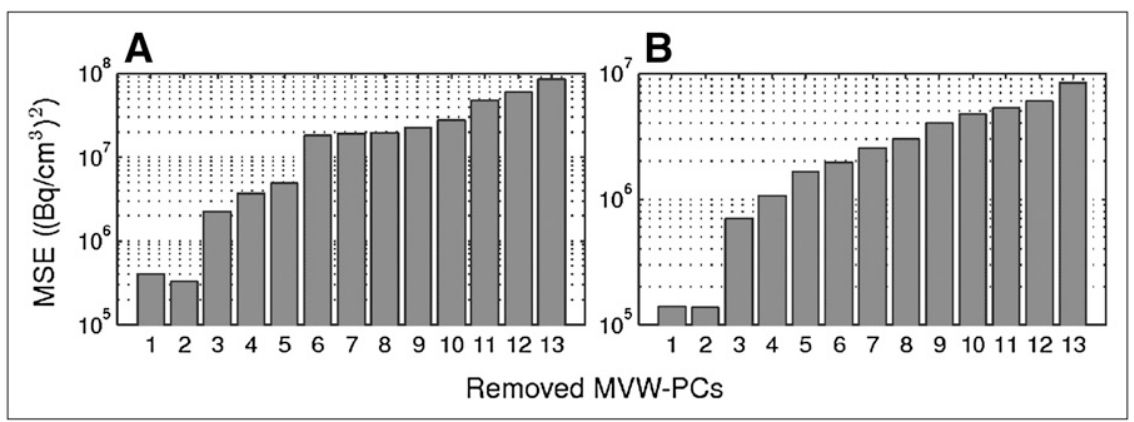

order PC noise prenormalization generated results closest to the results retrieved from the background noise prenormalization (for these specific datasets).

\section{Clinical Study for Evaluating Higher-Order PC Noise Prenormalization}

$\mathrm{MSE}_{\hat{x}}$, the MSE in comparison with the optimal component $\hat{x}$, for various reconstructions is shown in Figure 9. These bar graphs illustrate that the gland was optimally reconstructed using the first 3 masked volumewise PCs when higher-order PC noise prenormalization was applied, for both FBP and OSEM. The stomach was optimally reconstructed using the first 5 masked volumewise PCs with background noise prenormalization for FBP and using the first 6 masked volumewise PCs with standardized variable prenormalization for OSEM.

The differences between the prenormalizations are significant in the FBP data. The background noise prenormalization did not perform well on the dataset reconstructed with OSEM in the stomach VOI. Higher-order PC noise prenormalization performed fairly well on both the dataset reconstructed with FBP and the dataset reconstructed with OSEM.
The result from application of masked volumewise PCA with higher-order $\mathrm{PC}$ noise prenormalization on datasets reconstructed with FBP and OSEM is illustrated in Figure 10.

An example of a slice in a dimension-reduced dataset is shown in Figure 11. This dataset was reconstructed with OSEM and underwent higher-order PC prenormalization. It was then dimension-reduced to the first 5 masked volumewise PCs. The dimension-reduced dataset had a more homogeneous appearance, and the flickering of pixels that appears when several frames are viewed in a sequence was reduced.

The $\mathrm{MSE}_{\hat{x}}$ graphs in Figure 9 show that the removal-ofmean and the standardized-variable prenormalizations should be avoided when data reconstructed with FBP are analyzed.

\section{DISCUSSION}

The aim of this study was to reduce noise in dynamic PET datasets, reconstructed with either FBP or OSEM, using masked volumewise PCA.

The phantom study showed that, compared with FBP, OSEM had a higher expectation value in high-activity regions and a lower expectation value in regions where there

FIGURE 9. MSE in different reconstructions for different prenormalizations, compared with optimal signal $\hat{x}$. FBP is used in $A$ and $B$ whereas OSEM is used in $C$ and $D$. Measurements are from adrenal gland VOI (A and C) and stomach VOI (B and D). Index of higher-order PC corresponds to number of removed masked volumewise PCs in prenormalization step. Logarithmic scale is used.

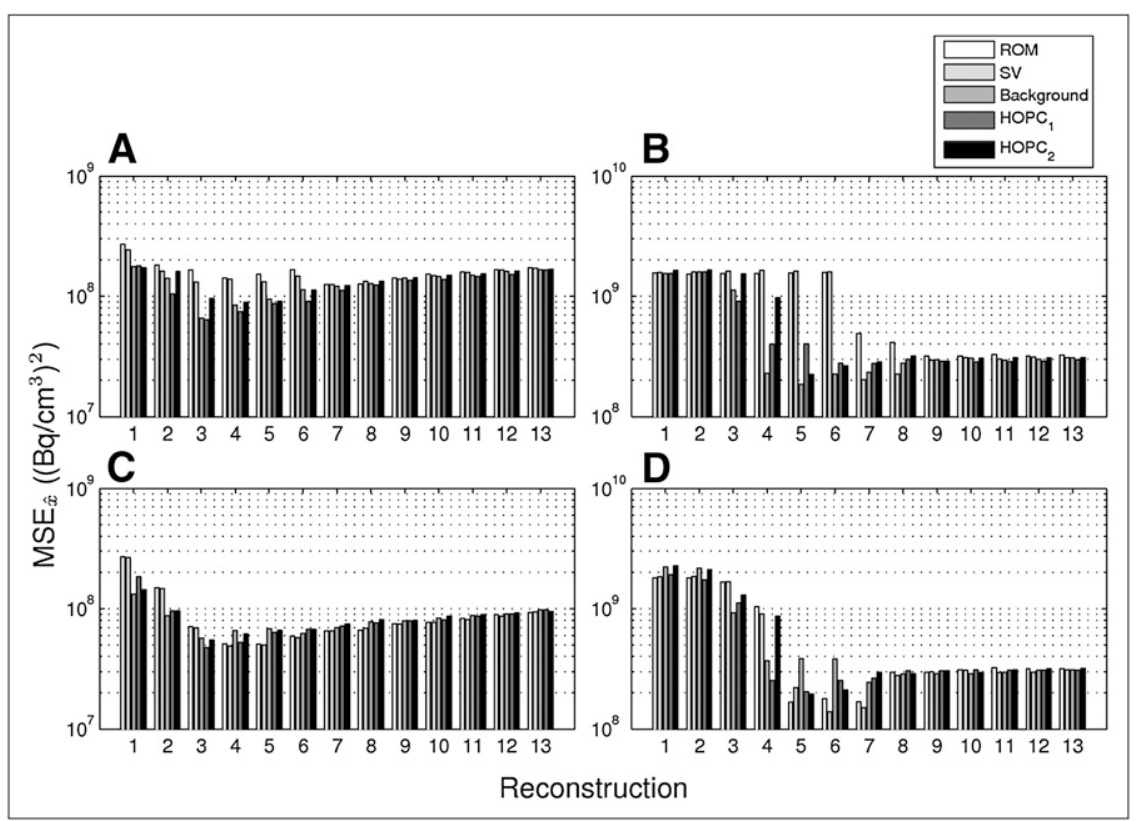


A

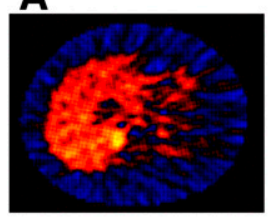

D

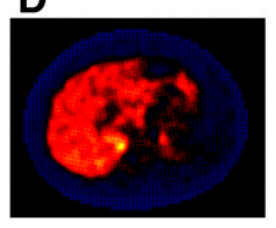

B

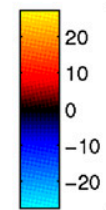

E

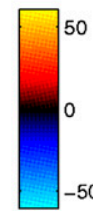

E
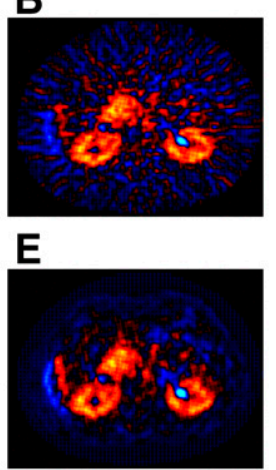

C
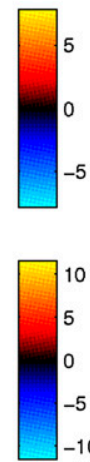

F
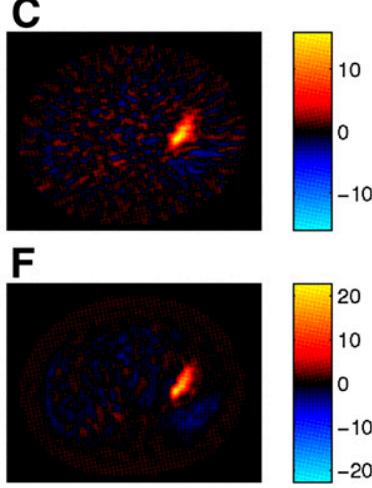

FIGURE 10. Slices from first 3 masked volumewise PCs from datasets reconstructed with FBP $(A-C)$ and OSEM (D-F). Most adrenal gland and general tracer behavior is described by masked volumewise PC 1 (A and D). Masked volumewise $P C 2$ ( $B$ and $E$ ) describes early tracer accumulation in kidneys, whereas tracer concentration in stomach can be separated with masked volumewise PC 3 (C and $F)$. was no activity. In high-activity regions, the SD of OSEM and FBP data was close to identical, whereas OSEM had an SD close to zero in regions without any activity. The trend toward an increased sample SD over time is due to the decay correction, which aims at keeping the expectation value constant at the expense of a higher SD. The 2 peaks that are visible in Figure 6 are probably due to the fact that the eXplore VISTA scanner has 2 separate detector rings.

The only parameter that has to be decided when higherorder PC prenormalization is performed is the number of lower-order masked volumewise PCs to remove to get a good estimate of the SD of the noise. Removal of the first, or the first 2, masked volumewise PCs gave scale factors close to the SD of the background noise in an ${ }^{11} \mathrm{C}$-Pittsburgh compound B and ${ }^{18}$ F-FDG study. If a large part of the signal is spanned by more than 2 masked volumewise PCs, it may be necessary to remove more masked volumewise PCs. Too much signal in the reconstruction will result in highly overestimated scale factors for the slices in which the signal is present, resulting in slices with low amplitude.

It is expected that, when used on OSEM data, background noise prenormalization would perform poorly because it is dependent on noise in the background and will not function at all if the background values are zero. Nevertheless, background noise prenormalization performed well in the adrenal gland region but poorly in the stomach region for OSEM data. Higher-order PC prenormalization showed great potential because it performed well on data reconstructed with both FBP and OSEM. None of the prenormalizations was superior to all the others on all datasets.

As Figure 9 illustrates, the MSE tends to decrease for every added masked volumewise PC up to a certain point, after which the error starts to increase again. The reason is that lower-order masked volumewise PCs describe mostly the signal whereas higher-order masked volumewise PCs describe noise rather than signal. The point after which the error starts to increase depends not only on the chosen prenormalization but also, even more, on the structure where the error is measured.

As seen in Figure 11, masked volumewise PCs are useful for drawing ROIs. Even though dimension reduction of input data in this study was used primarily as a tool to compare different prenormalizations, the method can be used on clinical data to reduce noise, as shown in Figure 11. One must be careful if small regions with small deviations in tracer behavior are of interest, or if the patient is moving during the scan, because this information tends to end up in higher-order masked volumewise PCs and be removed. Even though the image in Figure 11B looks low-pass-filtered, dimension reduction with PCA simply preserves the time-activity curves that are correlated and hence is independent of the spatial or temporal frequency of the signal.

Both higher-order PC and background noise prenormalization scales the dataset slice by slice. This procedure yields good results when there are clear differences in noise variance between different slices. However, one can assume
A

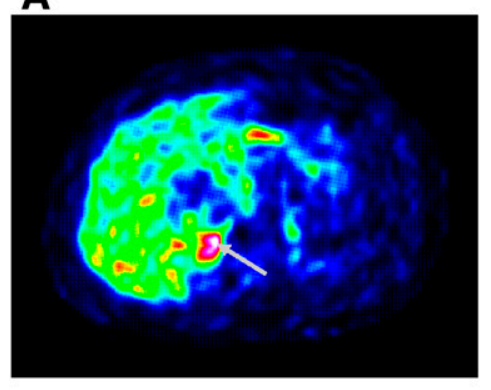

B

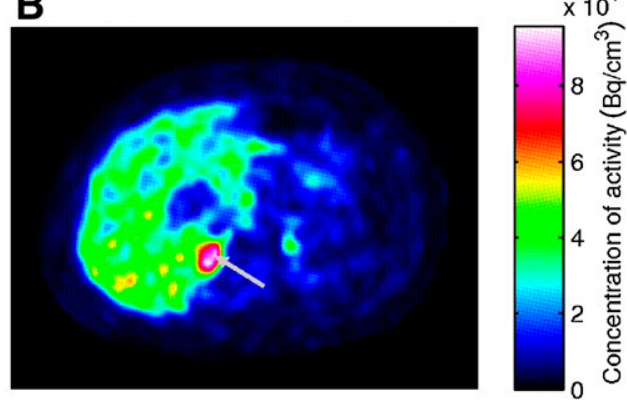

FIGURE 11. One slice in original (A) and dimension-reduced (B) dataset showing adrenal gland (arrows). 
that such is not the case when 3-dimensional reconstruction is used. Nor is this procedure as efficient for OSEM as for FBP (as shown in this study), because noise variance in datasets reconstructed with OSEM varies greatly within each slice depending on the signal. On the basis of this information, future studies aimed at increasing performance further by estimating the SD of each voxel and prenormalizing the dataset before PCA would be of interest.

\section{CONCLUSION}

Masked volumewise PCA of dynamic PET datasets reconstructed with FBP is much more dependant on an appropriate prenormalization than is analysis of data reconstructed with OSEM. This study showed that higherorder PC prenormalization can be used on dynamic datasets reconstructed with both methods. Higher-order PC prenormalization and dimension reduction with masked volumewise PCA, followed by reconstruction to the original frame space, shows promising results that can be applied in clinical trials.

\section{ACKNOWLEDGMENT}

We thank Prof. Anders Sundin for providing the clinical ${ }^{11} \mathrm{C}$-metomidate PET dataset used in this study. This study was financed by GEMS PET Systems AB and was partially carried out at Uppsala Applied Science Laboratory (UALF).

\section{REFERENCES}

1. Wrenn FR, Good ML, Handler P. The use of positron emitting radioisotopes for the localization of brain tumors. Science. 1951;113:525-527.

2. Razifar P, Axelsson J, Schneider H, Långström B, Bengtsson E, Bergström M. A new application of pre-normalized principal component analysis for improvement of image quality and clinical diagnosis in human brain PET studies: clinical brain studies using $\left[{ }^{11} \mathrm{C}\right]-\mathrm{GR} 205171,\left[{ }^{11} \mathrm{C}\right]-\mathrm{L}$-deuterium-deprenyl, $\left[{ }^{11} \mathrm{C}\right]-5$-hydroxy-Ltryptophan, $\left[{ }^{11} \mathrm{C}\right]-\mathrm{L}-\mathrm{DOPA}$ and Pittsburgh compound-B. Neuroimage. 2006;33: 588-598.

3. Razifar P, Hennings J, Monazzam A, Hellman P, Långström B, Sundin A. Masked volume wise principal component analysis of small adrenocortical tumours in dynamic $\left[{ }^{11} \mathrm{C}\right]$-metomidate positron emission tomography. BMC Med Imaging. 2009;9:6.

4. Wilson DW, Tsui BMW. Noise properties of filtered-backprojection and ML-EM reconstructed emission tomographic images. IEEE Trans Nucl Sci. 1993;40:11981203.

5. Wang Y, Seidel J, Tsui BMW, Vaquero JJ, Pomper MG. Performance evaluation of the GE Healthcare eXplore VISTA dual-ring small-animal PET scanner. $J$ Nucl Med. 2006;47:1891-1900.

6. Engbrant F, Monazzam A, Svensson PE, Olsson J, Bengtsson E, Razifar P. Signal extraction and separation in in vivo animal PET studies with masked volumewise principal-component analysis. J Nucl Med Technol. 2010;38:53-60.

7. Pearson K. On lines and planes of closest fit to points in space. Philos Mag. 1901;2:559-572.

8. Johnson RA, Wichern DW. Applied Multivariate Statistical Analysis. 3rd ed. Upper Saddle River, NJ: Prentice-Hall, Inc.; 1992. 